\title{
Developing a Scale for Quality of Life in Pediatric Oncology Patients Aged 7-12 - Children and Parent Forms
}

\author{
Asli Akdeniz Kudubes ${ }^{1}$, Murat Bektas ${ }^{2}$
}

\begin{abstract}
Background: This study was planned in an attempt to develop a scale for the quality of life in pediatric oncology patients aged 7-12, with child and parents forms. Materials and Methods: In collecting the study data, we used the Child and Parent Information Form, Visual Quality of Life Scale, Scale for Quality of Life Pediatric Oncology Patients Aged 7-12 and the Scale for the Quality of Life in Pediatric Oncology Patients Aged 7-12 for Parents. We also used Pearson correlation analysis, the Cronbach alpha coefficient, factor analysis and ROC analysis for the study data. Results: In this study, the total Cronbach alpha value of the parent form was 0.96, the total factor load being 0.54-0.90 and the total variance explained was $82.5 \%$. The cutoff point of the parent form was 93 points. The total Cronbach alpha value for the child form was 0.96 , with a total factor load of $0.55-0.91$ and the total variance being explained was $78.3 \%$. The cutoff point of the child form was 65 points. Conclusions: This study suggests that the Scale for Quality of Life in Pediatric Oncology Patients Aged 7-12 Child and Parents Forms are valid and reliable instruments in assessing the quality of life of children.
\end{abstract}

Keywords: Cancer - child - parents of children with cancer - quality of life

Asian Pac J Cancer Prev, 16 (2), 523-529

\section{Introduction}

Cancer is one of the important health problems in developed and under-developed countries. The number of cancer children and adults is gradually increasing in world population. While childhood cancers constitute approximately $0.5-1 \%$ of total cancers, this rate differs depending on age periods. It is estimated that the new case number will be 11.630 in children among 0-14 ages in America (American Cancer Society, 2013). Childhood cancers five-year survival now exceeds $70-80 \%$ (Vegian et al., 2012; Wiangnon et al., 2014). Of the cancers seen in a human being's lifetime, 1-2\% are diagnosed in children. In Turkey, 2500-3000 children under the age of 15 are newly diagnosed with cancer each year (Emir, 2009).

Quality of life in pediatric oncology is multidimensional. This condition include social, physical, and emotional execution functions of the child and family, but there are not limited. Measurement of quality of life, should be done from the perspective of the child and family and should be sensitive to changes occurring during the development (Bradly et al., 1996; Okcin and Karadakovan, 2012; Yakar and Pinar, 2013). Quality of life for children with cancer, physical and emotional problems, surgical interventions, radiotherapy, chemotherapy, prolonged hospitalization, side effects of the treatment, indoor and outdoor insulation, changes in the course of disease, child's position and role absence of loss within the family and society, disruption of school life, lack of support systems and coping methods are such as reasons decreased (Wong and Hockenberry, 2003; Kelly and Porock, 2005).

Cancer treatment is quite tired child and degradation of the balance physically and spiritually, it is a difficult process. Some of the problems can be prevent, reduced or controlled with careful assessment and appropriate interventions (Genc and Conk, 2008; Ainuddin et al., 2012; Arslan et al., 2013). In this regard have become important the cooperation between parents and nurses with family-centered approach (Bugge et al., 2008; Citak et al., 2013). Nurses should support and home care children with cancer and their families such as education, counseling, health assessment, facilitation of medical care, practice technical skills, providing emotional counseling child with cancer and family at the hospital and home care. This reason must determine quality of life of child (Wong and Hockenberry, 2003; Kelly and Porock, 2005; Demirbağ et al., 2013; Kudubes et al., 2014).

Evaluation of the quality of life in pediatric oncology patients is an important issue and there is a limited number of studies in this area. Even though there are various scales assessing the quality of life abroad, there is a limited number of studies analyzing the validity and reliability of these scales (Goodwin et al., 1994; Varni et al., 1998). In our country, there is not studies aimed at identifying the quality of life of pediatric oncology patients and the assessment of quality of life by the medical personnel 
which consequently disables the medical personnel and especially the nurses caring about children with cancer to identify the quality of life and plan the convenient interventions. In order to increase studies of quality of life in our country, we need more valid and reliable diseasespecific tools. Accordingly, this study aims to develop the Scale for the Quality of Life in Pediatric Oncology Patients Aged 7-12 Child and Parents Form.

\section{Materials and Methods}

\section{Population and sample}

The sample calculation that was required for the study was performed in the GPOWER statistical analysis program by taking the Type I error as 0.05 and Type II error 0.20 (80\% POWER), and in the study of Barrera, Pringle, Sumbler ve Saunder'ın (2000), the required sample size was determined as 91 by using the score averages. Another method being suggested for calculating the sample in scale development studies includes three rules as $5 \mathrm{~s}, 10 \mathrm{~s}$ and 100 s rule. It is emphasized that the researcher is required to include at least five individuals for each item in order to perform the factor analysis. It is also emphasized that there should be 10 individuals for each item unless there is a problem about reaching the sample (Sencan, 2005). In the study, we reached 204 children with cancer and their parents, who applied to a training and research hospital and a university hospital between 15 April-15 August 2014.

Inclusion criteria for the study were accepted as: 1) children aged 7-12 and diagnosed with cancer and their parents who were primarily responsible for their care, 2) Being literate, 3) Children and parents who were volunteer to participate in the study.

\section{Data collecting instruments}

Child and Parent Information Form: "Child and Parent Information Form" that is improved by basing on litterateur was consisted of 8 questions including the children's socio-demographic features, diagnosis, disease phase, treatments they received, period of receiving diagnosis, treatment period, ages of parents and sex (Collins et al., 2000; Woodgate and Degner, 2003; Woodgate et al., 2003).

Visual Quality of Life Scale-VQLS: "Visual Quality of Life Scale" is an assessment instrument that assesses the quality of life visually. It is graded between " 1 " and " 10 " and the increase of the scale score signifies the increase of the quality of life of the child.

Scale for the Quality of Life in Pediatric Oncology Patients Aged 7-12 - Child Form: The researcher examined the literature and reached general and child-specific scales regarding the quality of life. As a result of the literature review, dimensions were formed to determine the quality of life from all aspects (Aaronson ve ark., 1991; Goodwin et al., 1994; Varni et al., 1998). It consists of totally 32 items and 9. substance is reversed. Being a likert scale, this scale is graded between " 1 " and " 5 ". While the lowest score to be obtained from the scale is 32 , the highest score is 160 . The increase of the scale score signifies the increase of the quality of life of the child.

Scale for the Quality of Life in Pediatric Oncology
Patients Aged 7-12 - Parents Form: The researcher examined the literature and reached general and childspecific scales regarding the quality of life. As a result of the literature review, dimensions were formed to determine the quality of life from all aspects (Aaronson ve ark, 1991; Goodwin et al., 1994; Varni et al., 1998). It consists of totally 32 items and 9 . substance is reversed. Being a likert scale, this scale is graded between " 1 " and " 5 ". While the lowest score to be obtained from the scale is 32 , the highest score is 160 . The increase of the scale score signifies the increase of the quality of life of the child.

Being a likert scale, this scale is graded between " 1 " and " 5 ". While the lowest score to be obtained from the scale is 32 , the highest score is 160 . The increase of the scale score signifies the decrease of the quality of life of the child.

\section{Stages of the study}

The stages to be followed in developing the Quality of Life Scale and analyzing the validity and reliability are explained as follows; Stage of forming the item pool: An extensive examination should be made about the variable to be measured while designing the scale statements. The statements to be written should comprise all the ideational, affective and action-aimed elements involved in experiences regarding the variable to be measured or their dimensions that are required to be measured. As a consequence, the statements in the scale should constitute a sample that comprises the dimension of the measured and to-be-measured variable from all aspects and represents it (Akgul, 2003; Gozum and Aksayan, 2003; Ozdamar, 2005; Sencan, 2005; Simsek, 2007). While forming the item pool of the Quality of Life Scale for Pediatric Oncology Patients and Parents, we examined the literature and reached studies defining the quality of life and general and child-specific scales regarding the quality of life. As a result of the literature review, dimensions were formed to determine the quality of life from all aspects and item pools were developed for these dimensions (Aaronson et al., 1991; Goodwin et al., 1994; Varni et al., 1998).

Stage of forming the expert opinion: It is suggested to apply to at least ten expert opinions in order to determine the content validity of scales (Akgul, 2003; Gozum and Aksayan, 2003; Ozdamar, 2005; Sencan, 2005; Simsek, 2007). Fourteen expert opinions were received for the scales (ten academic members in the Department of Pediatric Health and Diseases Nursing, three academic members in the Department of Oncology Nursing and one academic member in the Department of Psychiatric Nursing). The experts were given the scale form and required to grade between 1-4 in order to assess the convenience of scale items ( $1=$ Requires a great change, $4=$ Very convenient). As a result of expert opinions, 10 items were excluded from the scale (I'm afraid of my disease recurrence, I'm scared about what will happen to me, I don't participate in fun activities, My father and my brothers would come to visit more often than I would be happy, Other people look at me disturbs me, I used to like to do things I do not want to do it anymore, Financial difficulties because of our failure to obtain what I want makes me sad, I'm having difficulty in collecting 
my attention, I'm having difficulty remembering things, I can not go to school in the hospital's happening), three item was added I feel reluctant to do something, Makes me sad constantly able to go to school, My favorite things (playing games, etc..) upset me that I can't), one item was revised (I have nausea and vomit) and the scale was used in its final form with 32 items.

Stage of forming the preliminary test: After receiving the expert opinions, it is suggested to apply the scale to a sample of 10-20 individuals, who have similar features with individuals to be measured, but are not involved in the sample (Akgul, 2003; Gozum and Aksayan, 2003; Ozdamar, 2005; Sencan, 2005; Simsek, 2007). The outline that was formed by receiving the expert opinions was applied to 25 children and their parents matching the scale sample criteria and since no negative feedback was received, it was decided to be applied to the larger group.

\section{Ethical issues}

The implementation of the research was started after 03.04.2014 dated and 1396-GOA protocol numbered resolution of Dokuz Eylul University Non-Invasive Research Ethics Committees. Institutional permissions were obtained in order to carry out the research. Besides, child and parents written and verbal permissions were obtained by meeting them and giving them information about the aim of the research.

\section{Analysis of the data}

In the data analysis, we used; the content validity analysis for the coherence analysis of descriptive statistics and expert opinions, Pearson's correlation analysis for the total item score analysis of scales and lower dimensions, Cronbach Alpha coefficient for the internal consistency of scales and lower dimensions, explanatory factor analysis for the item-factor relationship, $t$ test for the known group comparison and Pearson's correlation analysis for the relationship between the scale factors (Akgul, 2003; Gozum and Aksayan, 2003; Ozdamar, 2005; Sencan, 2005; Simsek, 2007). We used the ROC analysis in determining the cutoff point of the scale. The margin of error was taken as $\mathrm{p}=0.05$ in the assessment of the data.

\section{Results}

The children who participated in the study had an age average of $37.2+1.4 ; 55.5 \%$ were male, $48.5 \%$ female, $56.5 \%$ in the remission stage; $49 \%$ were diagnosed with leukemia, $51 \%$ received only chemotherapy, $25 \%$ chemotherapy-radiotherapy and surgical treatment, $18.1 \%$ received radiotherapy in the head-neck area and the average number of radiotherapy cures was $3.11+1.4$. Parents who participated in the study had an age average of $37.2+3.3$ and $82.4 \%$ were mothers.

Validity analyses

Content validity: Scores of fourteen experts were assessed with the content validity analysis and the coherence between expert scores was determined as 0.81 . The expert scores were observed to be coherent.

Construct validity: Construct Validity of the Parent Form: Construct validity of scales is tested through a number of different approaches. One of these approaches is the factor analysis. As a result of the factor analysis, the Kaiser-Meyer-Olkin coefficient (KMO) was determined as 0.777 and the Barlett test $\mathrm{X}^{2}=11846.400, \mathrm{p}=0.000$. The factor loads were determined as $0.54-0.90$. The total variance being explained is $82.5 \%$.

Construct Validity of the Child Form: As a result of the factor analysis, the Kaiser-Meyer-Olkin coefficient (KMO) was determined as 0.748 and the Barlett test $\mathrm{X}^{2}=10474.223, \mathrm{p}=0.000$. The factor loads were determined as $0.71-0.94$. The total variance being explained is $78.3 \%$.

Cutoff Point: One of the most effective methods being used in determining the optimum cutoff point is the Diagnostic index that is calculated through the values obtained from the ROC analysis and the Youden index (Perkin and Schisterman, 2005). The value of the Youden index varies between -1 and +1 and it is indicated that the closer it is to +1 , the greater the power of distinguishing becomes (Sencan, 2005). The scale score where these two indexes obtain the highest value and coincide determines the optimum cutoff point for that scale (Perkin and Schisterman, 2005).

Table 1 shows the values of Diagnostic index (DI) and Youden index (YI) that were calculated as a result of the ROC analysis, which was performed to determine the cutoff point. Especially YI is defined as the point that is closest to +1 where the best distinction could be made and it is suggested to determine the cutoff point through the comparison with the point where DI obtains the highest value (Perkin and Schisterman, 2005; Sencan, 2005). We determined 65 points, where the child form obtains the highest DI and YI values, as the cutoff point and measured the sensitivity of the scale as 0.50 and specificity 087 at this point and those who obtained 64.9 and below were evaluated as low quality of life of children (Table 1). We determined 93 points, where the parent form obtains the highest DI and YI values, as the cutoff point and measured the sensitivity of the scale as 0.91 and specificity 0.68 at this point and those who obtained 92.9 and below were evaluated as low quality of life of children (Table 1 ).

Table 1. Cutoff Point, Prediction Values and Values of the Area under the Curve (AUC) in Predicting the State of Quality of Life in the ROC Analysis of the Child and Parent Form

The known group comparison: One of the methods being used in determining the construct validity of scales is the known group comparison (Erkus, 2002; Gozum and Aksayan, 2003). In this analysis, a significant difference is expected between the quality of life score averages of children with and without quality of life according to the cutoff points. According to the parent form, we determined the score average of those with quality of life as $81.6+$ 19.7 and the score average of those without quality of life as $120.0+13.9$. According to the state of quality of life, a statistically significant difference was determined between the score averages of the parent form $(t=12.476, p=0,000)$.

According to the child form, we determined the score average of those with quality of life as $56.1+10.9$ and the score average of those without quality of life as $74.5+$ 7.7. According to the state of quality of life, a statistically significant difference was determined between the score 
Table 1. Cutoff Point, Prediction Values and Values of the Area under the Curve (AUC) in Predicting the State of Quality of Life in the ROC Analysis of the Child and Parent Form

\begin{tabular}{lccccccc}
\hline & Cut Point & Sensitivity & Specificity & $\mathrm{p}$ & EAA (\% 95 Confidence Interval) & Youden İndex & Diagnostic İndex \\
\hline Child Form & 65 & 0.50 & 0.87 & 0.000 & $0.760(0.689-0.832)$ & 0.378 & 1,378 \\
Parents Form & 93 & 0.91 & 0.68 & 0.000 & $0.748(0.636-0.859)$ & 0.260 & 1,260 \\
\hline
\end{tabular}

Table 2. Cronbach Alpha Coefficient and Reliability Analysis of the Fatigue Scale for Parents and Child Form

\begin{tabular}{lcccccc}
\hline Quality of Life Scale & Cronbach Alpha $(\alpha)$ & Mean & SD & Floor Effect $\%$ & Ceiling Effect $\%$ & Skewness \\
\hline Parents Form & 0.96 & 86.9 & 23.2 & 0.5 & 0 & -0.413 \\
Child Form & 0.96 & 60.0 & 12.7 & 3.4 & 0.0 & -0.438 \\
\hline
\end{tabular}

averages of the parent form $(\mathrm{t}=12.476, \mathrm{p}=0,000)$.

\section{Reliability analyses}

Reliability Analysis of the Parents and Child Form: The reliability coefficients of the child and parent form were determined $\alpha=0.96$ in total for the scale (Table 2).

Quality of Life Scale for Parents Form mean score was $86.9+23.2$, floor and ceilinf effect were $0.5 \%$ and $0.0 \%$, respectively, skewness was -0.413 (Table 2).

Quality of Life Scale for Child Form mean score was $60.0+12.7$, floor and ceilinf effect were $3.4 \%$ and $0.0 \%$, respectively, skewness was -0.438 (Table 2 ).

Table 2. Cronbach Alpha Coefficient and Reliability Analysis of the Quality of Life Scale for Parents And Child Form

Total Item Score Correlations of the Parent Form and Test-Retest Correlations of Items: Examining the item-total score correlations of the scale consisting of 32 items for the reliability study, it was determined that the correlation coefficients of the scale between 0.38-0.99 $(\mathrm{p}=0.000)$.

Besides, examining the correlation between the first and second application scores of each item, it was determined that the test-retest reliability coefficient of only two items were on margin $(r=0.120,0.151)$, and the testretest reliability coefficients of other items were between $\mathrm{r}=0.27-0.93$ and statistically significant $(\mathrm{p}=0.000)$.

Total Item Score Correlations of the Child Form and Test-Retest Correlations of Items: Examining the item-total score correlations of the scale consisting of 32 items for the reliability study, it was determined that the correlation coefficients of the scale between 0.38-0.99 $(\mathrm{p}=0.000)$.

Besides, examining the correlation between the first and second application scores of each item, it was determined that the test-retest reliability coefficient of only one items were on margin ( $\mathrm{r}=0.140)$, and the test-retest reliability coefficients of other items were between $r=$ $0.28-0.95$ and statistically significant $(\mathrm{p}=0.000)$.

\section{Test-Retest Reliability of the Child and Parent Form (Stability)}

After applying the parent form for twice every three weeks, we assessed the stability, in other words the testretest reliability coefficient of the scale with the Pearson Product-Moment Correlation Coefficient. A positive and statistically significant relationship was determined between the test-retest score averages of the scale ( $\mathrm{r}=$ 0.837, $\mathrm{p}=0$.000).

After applying the child form for twice every three weeks, we assessed the stability, in other words the testretest reliability coefficient of the scale with the Pearson Product-Moment Correlation Coefficient. A positive and statistically significant relationship was determined between the test-retest score averages of the scale ( $\mathrm{r}=$ $0.835, \mathrm{p}=0.000)$.

Besides, we conducted the $t$ test for dependent groups in order to determine whether there was a difference between the score averages obtained from the scale as a result of two measurements that were applied every three weeks; however, we determined no statistically significant difference between the score averages $(\mathrm{p}>0.05)$.

\section{Relationship between the Study Variables of the Child and Parent Form}

We assessed the relationship between the variables with the Pearson correlation analysis and determined a statistically significant relationship between the parent form and child form at a level of $r=0.988$, between the parent form and parent VAS score at a level of $r=0.677$ and the child VAS score at a level of $r=0.677(p<0.01)$.

\section{Discussion}

If an instrument will be used in a different language it is necessary to show that it has the same validity and reliability as the instrument's original format (Savasır and Sahin, 2002; Gozum and Aksayan, 2003; Sencan, 2005). For this reason it was necessary to evaluate the validity and reliability of the Parents and Child Form, which will be used in a Turkish sample.

Being prepared to determine the content validity, the scale is examined by experts and reviewed and reprepared according to criticisms (Ozguven, 2000; Sencan, 2005). It is possible to use a form that would enable the experts to evaluate the coherence of items through giving points. Consensus of the majority of experts may be accepted as an indicator for the content validity (Gozum and Aksayan, 2003; Sencan, 2005). In this study, we received fourteen expert opinions in order to assess the coherence of the items of the parent and child form for the language and culture. We also assessed the suggestions of experts regarding the expression and content of items, excluded some of the items and changed the statementsof 
one item. In the content validity analysis, minimum values regarding the number of experts also signify the statistical significance of the item. In the content validity analysis, the minimum value for fourteen experts is 0.51 at a significance level of $\mathrm{p}=0.05$ (Yurdugul, 2005). We assessed the scores of fourteen experts with the content validity analysis and determined the coherence between the expert scores as 0.81 . The expert scores were observed to be coherent. According to these results, it is possible to assert that the statements of the parent and child form are convenient for the Turkish culture and represent the area to be measured, and the content validity is ensured.

Factor and explanatory factor analyses: One of the main objectives of the factor analysis is to reveal some new structures by using the relationships between variables. In other words, it is aimed to form common factors by grouping the variables in the factor analysis (Tavsanel, 2002; Gozum and Aksayan, 2003; Sencan, 2005). As a result of the factor analysis in this study, the KaiserMeyer-Olkin coefficient (KMO) of the parent form was determined as 0.777 and the Barlett test $\mathrm{X}^{2}=11846.400$, $\mathrm{p}=0.000$. These values showed that the number of samples was convenient for a factor analysis. The factor loads were determined as $0.54-0.90$ for the scale. The total variance being explained is $82.5 \%$.

As a result of the factor analysis in this study, the Kaiser-Meyer-Olkin coefficient (KMO) of the child form was determined as 0.863 and the Barlett test $\mathrm{X} 2=111176.579, \mathrm{p}=0.000$. These values showed that the number of samples was convenient for a factor analysis. The factor loads were determined as $0.55-0.91$ for the scale. The total variance being explained is $78.3 \%$.

The factor structure of the scale becomes stronger as the rate of the obtained variance is higher. In the studies, the variance rates between $40-60 \%$ are accepted as sufficient (Tavsenel, 2002; Sencan, 2005). In this study, on the other hand, we obtained a high and sufficient total variance through obtaining a variance that could be explained at a rate of $80 \%$ in both scales. As a result of the analysis, it was suggested that the parent and child form had a coherent construct validity.

As a result of the ROC analysis that was performed to determine the cutoff point, we determined 93 points, where the sensitivity was the highest and the specificity was the lowest in the parent form, as the cutoff point and detected the sensitivity of the scale as 0.91 and the specificity 0.68 at this point (Table 1 ). Those who obtained 92.9 and below from the parent form were evaluated as low quality of life of children. As a result of the ROC analysis that was performed to determine the cutoff point, we determined 65 points, where the sensitivity was the highest and the specificity was the lowest in the child form, as the cutoff point and detected the sensitivity of the scale as 0.50 and the specificity 0.87 at this point (Table 1). Those who obtained 64.9 and below from the child form were evaluated as low quality of life of children. The ROC curve gives a coherent cutoff point for the assessment instrument and the decisions that are made according to this cutoff enable us to obtain the sensitivity and specificity rates. While Sensitivity is shortly defined as "the condition where those who are sick in reality are also sick according to the cutoff point that is taken during the test", the Specificity is defined as "the condition where those who are healthy in reality are also found healthy as a result of the test". The curve moves upward (high sensitivity area) and to the left (low false positive rate area) as the test becomes better. If the area under the ROC curve (AUC) is 0.5 , there is no distinction, if between 0.5 and 0.7 , the power of distinguishing the test is statistically insignificant, if between 0.7 and 0.8 , it is acceptable, if between 0.8 and 0.9 , it is very good and if above 0.9 , it is excellent (Dirican, 2001). Accordingly, it is seen that the EAA of the parent form is between 0.636-0.859 and has a very good level of distinction, and it also has the ability of significantly distinguishing the children with and without low quality of life (Table 1). On the other hand, the EAA of the child form is between $0.689-0.832$ and has a very good level of distinction, and it also has the ability of significantly distinguishing the children with and without low quality of life (Table 1).

One of the methods being used in determining the construct validity of scales is the group comparison (Erkus, 2002; Gozum and Aksayan, 2003). In this analysis, a significant difference is expected between the quality of life averages of children with and without low quality of life. In this study, we determined the quality of life states of children according to the cutoff point with the help of the parent form and assessed those with a score of 92.9 and below as low quality of life of children and those with a score of 93 and above as non- low quality of life of children. As a result of the analysis, a significant difference was determined between the scale score averages of children with and without low quality of life in the parent form $(t=9.851, p=0,000)$. The presence of the difference not only indicates that the parent form could significantly determine the low quality of life of children, but also reveals the construct validity of the scale (Erkus, 2002; Gozum and Aksayan, 2003). This study determined the quality of life of children according to the cutoff point with the help of the child form and assessed those with a score of 64.9 and below as low quality of life of children and those with a score of 65 and above as non- low quality of life of children. As a result of the analysis, a significant difference was determined between the scale score averages of children with and without low quality of life in the child form $(t=12.476, p=0,000)$. The presence of the difference not only indicates that the child form could significantly determine the low quality of life children, but also reveals the construct validity of the scale (Erkus, 2002; Gozum and Aksayan, 2003).

Ranking the responses to items, the Likert attitude scales calculate the Cronbach alpha coefficient as an indicator of homogeneity. This test shows not only the internal consistency, but also whether the items measure the same feature and whether they are related with the subject to be measured or not. The reliability coefficient in an assessment instrument should be close to 1 as much as possible (Tavsanel, 2002; Gozum and Aksayan, 2003; Sencan, 2005). The reliability coefficients of the parent form were determined as $\alpha=0.96$ (Table 2). The reliability coefficients of the child form were determined as; $\alpha=0.96$. It was observed that the internal consistency of the scale 
had a high level of reliability (Table 2). Both the scale and its lower dimensions had a Cronbach alpha coefficient above 0.90 , which shows that the scale has a very good reliability (Gozum and Aksayan, 2003).

There are different methods that are followed in selecting items in the scale development studies. One of these methods is used in assessing the item total score correlations of scale items and in excluding the items with lower correlation values from the scale. The value to be used in the item selection is suggested to be between 0.20 0.25 and above. Highness of the correlation coefficient is accepted as an indicator of the coherence of that item for the theoretical structure being measured (Dirican, 2001; Erkus, 2002; Gozum and Aksayan, 2003).

Examining the item-total score correlations of the parent form consisting of 32 items for the reliability study, it was determined that the correlation coefficients of the scale (Pearson Product-Moment Correlation Coefficient) were statistically significant between $0.38-0.99(\mathrm{p}=0.000)$. Examining the item-total score correlations of the child form consisting of 32 items for the reliability study, it was determined that the correlation coefficients of the scale (Pearson Product-Moment Correlation Coefficient) were statistically significant between $0.38-0.99(\mathrm{p}=0.000)$. It is observed that the items in the scale are compatible with the theoretical structure of the scale and provide a sufficient correlation. Item-total score analysis is accepted as an indicator of not only the reliability, but also the validity (internal consistency) and it reflects the construct validity of the scale (Erkus, 2002).

Assessing the Coherence of the Lower Dimensions of the Scale between the Test-Retest Score Averages with the Help of the Correlation Analysis and t Test

Test-retest measurements are among the most frequently used reliability analyses assessing the stability of the assessment instrument. They are generally assessed by conducting the Pearson Product-Moment Correlation analysis (Gozum and Aksayan, 2003; Sencan, 2005). Being caculated to determine the stability (consistency) of an assessment instrument against time, the correlation coefficient is accepted to have a higher reliability as it gets closer to +1 . In instruments, the correlation coefficient between the test-retest scores is suggested to be at least 0.70 (Gozum and Aksayan, 2003; Sencan, 2005). In this study, we determined the stability coefficient of two applications of the parent form, which was repeated every three weeks, as $0.837(\mathrm{p}=0.000)$. On the other hand, the stability coefficient of two applications of the child form, which was repeated every three weeks, was determined as 0.835 ( $\mathrm{p}=0.000)$. It was observed that the parent and child form had a high reliability and the results were similar in first measurements and in repeated measurements.

Even if the test-retest correlation coefficient is sufficient, it is suggested to examine the score averages and standard deviations of two measurement results and have similar measurement results (Gozum and Aksayan, 2003; Sencan, 2005). For that purpose, we examined whether there was a difference between the results that were obtained from the scale being applied every three weeks with the help of the "t test for dependent groups" and determined no statistically significant difference between the score averages $(\mathrm{p}>0.05)$. Since individuals had similar and consistent responses to the items of the assessment instrument and the instrument proved to be stable when it was applied to them in different times (Tavsanel, 2002; Gozum and Aksayan, 2003; Sencan, 2005), the child and parent form were observed to be highly reliable.

Even though there may not be a significant difference between the total scores of individuals, they may give different answers to each item. Thus, it is required to also consider the consistency between the items in both applications (Tavsanel, 2002; Gozum and Aksayan, 2003; Sencan, 2005). Examining the correlation between the first and second application scores of each item, it was determined that the test-retest reliability coefficients of only two items of the parent form were on margin (8.item $=0.120$ ve 10. item $=0.151)$ and the test-retest reliability coefficients of other items were between $r=$ $0.27-0.93$ and statistically significant $(p=0.000)$. On the other hand, examining the correlation between the first and second application scores of child form of each item, it was determined only the test-retest reliability coefficient of one item is low ( 8 .item $=0.140)$, other items the test-retest reliability coefficients of the child form were between $\mathrm{r}=0.28-0.95$ and statistically significant $(\mathrm{p}=0.000)$. Items in the child and parent form gave similar results in both measurements, which signifies that the items are comprehensible and they measure consistently.

It is observed that the relationship between the two variables becomes stronger as it gets closer to 1 (Tavsanel, 2002; Gozum and Aksayan, 2003; Sencan, 2005). The relationship between the variables was assessed with the Pearson correlation analysis and it was found to be high and statistically significant between the scores of the parent form and child form $(r=0.988)$, between scores of the parent form and parent visual quality of life scale ( $\mathrm{r}=$ 0.677 ) and between the scores of the child form and child visual quality of life scale $(\mathrm{r}=0.677)(\mathrm{p}<0.01)$. A high and significant relationship is observed between the scores of the child and parent form and between the scale scores and visual quality of life scale quality of life scale scores. A high level of relationship signifies that the scales measure similar things and they measure them accurately. This result reveals that the scales are both valid and reliable.

There is no sufficient number of scales regarding the quality of life identification that are developed to be used in children aged 7-12 and analyzed in terms of validity and reliability abroad. In our country, seen that isn't presence of a scale to diagnose the quality of life for children with cancer. This number is apparently insufficient. Thus, the scale for quality of life in children and parents that was developed in this study is a convenient and comprehensive scale for our hospitals as it not only identifies the quality of life of children aged 7-12, but also is peculiar to our country. Besides, a great majority of children and parents in the study sample were in regional hospitals receiving patients from every region of Turkey, which supports the generalizability of the scale.

In conclusion, it is suggested to apply this scale, which was developed as there was no sufficient number of valid/ reliable identification instruments for pediatric patients in 
Turkey, in pediatric oncology clinics and to reassess the usage outcomes in the long term.

There is a need for valid-reliable instruments to manage the quality of life of pediatric oncology patients and to determine and apply the required nursing interventions. This study suggests that the Scale for the Quality of Life in Pediatric Oncology Patients Aged 7-12 Child and Parents Form are valid and reliable instruments in measuring the quality of life of children. This instrument is convenient for professionals to prevent and manage the quality of life. Professionals could develop interventions for children and parents concerning the results obtained from this scale.

\section{References}

Aaronson NK, Ahmedzai S, Bullinger M, et al (1991). The EORTC core quality of life questionnaire: Interim results of an international field study. In: Osoba D. ed. Effect of Cancer on Quality of Life. Boca Raton, FL: CRC Press:185-203.

Ainuddin HA, Loh SY, Low WY, et al (2012). Quality of life of multiethnic adolescents living with a parent with cancer. Asian Pac J Cancer Prev, 13, 6289-94.

American Cancer Society (2013). Access Date: 15.10.2013. http://www.cancer.org/acs/groups/content/@ epidemiologysurveilance/documents/document/ acspc-036845.pdf.

Akgul A (2003). Statistical analysis techniques, using SPSS in medical research. Ankara: Offset Labour Limited Company, 187-224, 382-5.

Arslan FT, Basbakkal Z, Kantar M (2013). Quality of life and chemotherapy-related symptoms of Turkish cancer children undergoing chemotherapy. Asian Pac J Cancer Prev, 14, 1761-8.

Barrera M, Boyd-Pringle LA, Sumbler K, et al (2000). Quality of life and behavioral adjustment after pediatric bone marrow transplantation. Bone Marrow Transplantation, 26, 427-35.

Bradlyn AS, Ritchey AK, Harri, CV, et al (1996). Quality of life research in pediatric oncology. American Cancer Society, 1333-9.

Bugge KE, Helseth S, Darbyshire P (2008). Children's experiences of participation in a family support program when their parent has incurable cancer. Cancer Nursing, 31, 427-34.

Collins JJ, Byrnes ME, Dunkel IJ (2000). The measurement of symptoms in children with cancer. J Pain Symptom Manage, 19, 363-77.

Citak EA, Toruner EK, Gunes NB (2013). Exploring communication difficulties in pediatric hematology: Oncology nurses. Asian Pac J Cancer Prev, 14, 5477-82.

Demirbağ BC, Kurtuncu M, Guven H (2013). Knowledge of Turkish mothers with children in the 0-13 age group about cancer symptoms. Asian Pac J Cancer Prev, 14, 1031-5.

Dirican A (2001). Evaluation and comparison of diagnostic test performance. J Cerrahpasa Medical, 32, 25-30.

Emir S (2009). Childhood cancers and how we can protect our children against cancer. Pediatrics Bulletin, 6, 2-8.

Erkus A (2002). Psychometrics is overwritten. Turkish Psychological Association Publication, Ankara, 1-30.

Genc ER, Conk Z (2008). Impact of effective nursing interventions to the fatigue syndrome in children who receive chemotherapy. Cancer Nursing, 31, 312-7.

Goodwin DAJ, Boggs SR, Graham-Pole J (1994). Development and validation of the pediatric oncology quality of life scale. J Psychol Assess, 6, 321-8,

Gozum S, Aksayan S (2002). Guidelines for cross-cultural scale adaptation II: Psychometric properties and cross-cultural comparison. J Nur Res Development, 5, 3-14.

Hinds PS, Hockenberry-Eaton M (2001). Developing a research program on fatigue in children and adolescents diagnosed with cancer. J Pediatr Oncol Nurs, 18, 3-12.

Karasar N (1999). Scientific research method. Ankara: Memories Publishing.

Kelly KP, Porock D (2005). A survey of pediatric oncology nurses' perceptions of parent educational needs. $J$ Pediatr Oncol Nurs, 22, 58-66.

Kudubes AA, Bektas M, Ugur O (2014). Symptom frequency of children with cancer and parent quality of life in Turkey. Asian Pac J Cancer Prev, 15, 3487.

Matzioua V, Perdikarisb P, Felonic D, et al (2008). Cancer in childhood: Children's and parents' aspects for quality of life. Eur J Oncol Nur, 12, 209-16.

Okcin F, Karadakovan A. (2012). Reliability and validity of the Quality of Life-Family Version (QOL-FV) in Turkish family caregivers of patients with cancer. Asian Pac J Cancer Prev, 13, 4235-840.

Ozdamar K (2005). Software Packages with Statistical Data Analysis. Eskisehir: Kaan Bookstores, 235-78.

Ozguven IE (2000). Psychologic tests. Ankara: System Offset.

Perkins NJ, Schisterman EF (2005). The Youden Index and the optimal cut point corrected for measurement error. Biom J, 47, 428-41.

Savasır I, Sahin HN (2002). Evaluation in cognitive behavioral therapy: Frequently used tools. Ankara: Turkish Psychological Association Publication.

Sencan H. (2005). The reliability and validity of the Social and Behavioral Measurement. Ankara: Seckin Publishing.

Simsek OF (2007). Structural equation modeling inputs: basic principles and application of LISREL. İstanbul: Ekinoks Publishing.

Tavsanel E (2002). Attitudes measurement and data analysis with SPSS. Ankara: Nobel Publishing House, 16-61.

Wiangnon S, Jetsrisuparb A, Komvilaisak P, et al (2014). Childhood cancer incidence and survival 1985-2009, Khon Kaen, Thailand. Asian Pac J Cancer Prev, 15, 7989-93.

Woodgate RL, Degner LF (2003). Expectations and beliefs about children's cancer symptoms: perspectives of children with cancer and their families. Oncol Nurs Forum, 30, 479-91.

Woodgate RL, Degner LF, Yanofsky R (2003). A different perspective to approaching cancer symptoms in children. $J$ Pain Symptom Manage, 26, 800-17.

Wong DL, Hockenberry MJ (2003). Wong's nursing care of infants and chidren, USA: Mosby Inc, 905-74, 1584-1635.

Varni JW, Katz ER, Seid M, et al (1998). The Pediatric Cancer Quality of Life Inventory (PCQL) Instrument development, descriptive statistics, and cross-informant variance. J Behav Medicine, 21, 179-204.

Vegian LH, Lubin JH, Anderson H, et al (2012). A pooled analysis of thyroid cancer incidence fallowing radiotherapy for childhood cancer. Radiat Res, 178, 365-76.

Yakar HK, Pinar R (2013). Reliability and validity of Turkish version of the caregiver quality of life index cancer scale. Asian Pac J Cancer Prev, 14, 4415-19. 